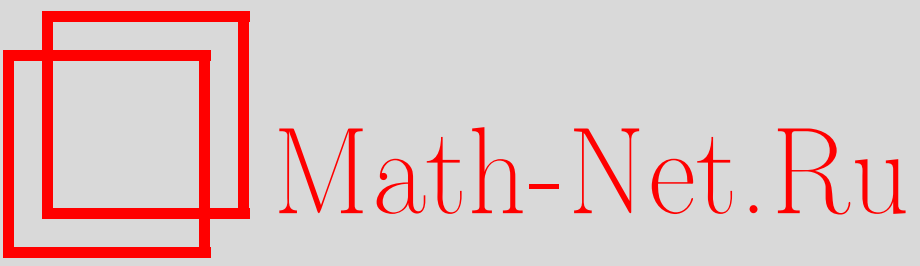

В. А. Кыров, Решение задачи вложения для двумерных и трехмерных геометрий локальной максимальной подвижности, Итоги науки и техн. Сер. Соврем. мат. и ее прил. Темат. обз., 2021, том 194, 124-143

DOI: https://doi.org/10.36535/0233-6723-2021-194-124-143

Использование Общероссийского математического портала Math-Net.Ru подразумевает, что вы прочитали и согласны с пользовательским соглашением

http: //www.mathnet.ru/rus/agreement

Параметры загрузки:

IP: 18.234 .156 .22

26 апреля 2023 г., 15:32:07 


\title{
РЕШЕНИЕ ЗАДАЧИ ВЛОЖЕНИЯ ДЛЯ ДВУМЕРНЫХ И ТРЕХМЕРНЫХ ГЕОМЕТРИЙ ЛОКАЛЬНОЙ МАКСИМАЛЬНОЙ ПОДВИЖНОСТИ
}

\author{
(c) 2021 г. $\quad$ B. А. КЫРОВ
}

\begin{abstract}
АннотАция. В современной геометрии большое значение имеет изучение геометрий максимальной подвижности. Некоторые из таких геометрий изучены хорошо (геометрии Евклида и Лобачевского, псевдоевклидова, симплектическая, сферическая геометрия и др.), в то время как другие (гельмгольцевы, псевдогельмгольцевы и др.) еще не привлекали активного внимания исследователей. Полной классификации геометрий максимальной подвижности пока нет. В данной работе приведены результаты решения классификационной задачи для двумерных и трехмерных геометрий локальной максимальной подвижности. Эта задача решается методом вложения в классе аналитических функций и сводится к решению функциональных уравнений специального вида.

Ключевые слова: геометрия максимальной подвижности, группа движений, функциональное уравнение.
\end{abstract}

\section{SOLUTION OF THE EMBEDDING PROBLEM FOR TWO-DIMENSIONAL AND THREE-DIMENSIONAL GEOMETRIES OF LOCAL MAXIMUM MOBILITY}

\section{(C) 2021 V. A. KYROV}

\begin{abstract}
In modern geometry, the study of the geometries of maximum mobility is of great importance. Some of these geometries are well studied (for example, the Euclidean and Lobachevsky geometries, pseudo-Euclidean, symplectic, spherical geometry, etc.), while others (for example, Helmholtz and pseudo-Helmholtz geometries) have not yet attracted active attention of researchers. There is still no complete classification of the geometries of maximum mobility. In this work, we present some results concerning the classification problem for two- and three-dimensional geometries of local maximum mobility. This problem is reduced to functional equations of a special form and is solved by the embedding method in the class of analytic functions.
\end{abstract}

Keywords and phrases: geometry of maximum mobility, motion group, functional equation.

AMS Subject Classification: 53D05,39B22

1. Введение. Для современной математики большое значение имеет изучение геометрий максимальной подвижности. $\mathrm{K}$ таким геометриям относятся: геометрия Евклида, псевдоевклидова геометрия, сферическая геометрия, геометрия Лобачевского и др. Полной же классификации геометрий максимальной подвижности не существует. Имеются отдельные классификации, в частности, классификация Терстона трехмерных максимально односвязных геометрий, допускающих компактные фактор-геометрии (см. $[1,17])$. Эта классификация специальных геометрий глобальной максимальной подвижности содержит в себе следующие геометрии:

(1) трехмерные геометрии с постоянной секционной кривизной: $\mathbb{R}^{3}, S^{3}, H^{3}$; 
(2) произведение пар геометрий: $S^{2} \times \mathbb{R}, H^{2} \times \mathbb{R}$;

(3) геометрии на группах Ли Nil, Sol, $\widetilde{S L}_{2}$ с некоторыми левоинвариантными метриками. Отметим, что группы движений геометрий из списка Терстона имеют размерность $\leqslant 6$.

В данной рассмотрены геометрии локальной максимальной подвижности, т.е. случай, когда группа движений имеет максимальную размерность, равную $n(n+1) / 2$ для $n$-мерной геометрии. Г. Г. Михайличенко построил полную классификацию таких двумерных геометрий (см. $[13,16])$, a В. Х. Лев-классификацию трехмерных геометрий (см. [12]). Эти классификации построены в рамках теории феноменологически симметричных геометрий и содержат как ранее известные геометрии (геометрия Евклида, псевдоевклидова геометрия, Лобачевского, симплектическая и т. д.), так и неизвестные геометрии (геометрия Гельмгольца, симплициальные геометрии, особые расширения евклидовой геометрии и т. д.). Феноменологически симметричные геометрии по сути и являются геометриями локальной максимальной подвижности и задаются функциями пары точек $f(A, B)$, где $A$ и $B$-точки многообразия $M$ (см. $[13,16])$. Поясним суть термина «феноменологическая симметрия». Феноменологическая симметрия, например, на двумерном многообразии означает существование функциональной связи между шестью значениями функции $f$ для произвольных четырех точек $\langle A, B, C, D\rangle$ этого многообразия: $\Phi(f(A, B), f(A, C), f(A, D), f(B, C), f(B, D), f(C, D))=0$, где $\Phi$ - некоторая дифференцируемая функция. Отметим, что для геометрии Евклида, задаваемой функцией пары точек

$$
f(A, B)=\left(x_{A}-x_{B}\right)^{2}+\left(y_{A}-y_{B}\right)^{2},
$$

такая функциональная связь записывается через определитель Кэли-Менгера:

$$
\left|\begin{array}{ccccc}
0 & 1 & 1 & 1 & 1 \\
1 & 0 & f(A, B) & f(A, C) & f(A, D) \\
1 & f(A, B) & 0 & f(B, C) & f(B, D) \\
1 & f(A, C) & f(B, C) & 0 & f(C, D) \\
1 & f(A, D) & f(B, D) & f(C, D) & 0
\end{array}\right|=0 .
$$

Подобные функциональные связи найдены также для псевдоевклидовой геометрии, симплектической геометрии, геометрии Лобачевского и т. д. Не для всех феноменологически симметричных геометрий известны явные записи функциональной связи.

Г. Г. Михайличенко доказал теорему об эквивалентности феноменологической и групповой симметрий, согласно которой вместо феноменологической симметрии $n$-мерной геометрии можно говорить о групповой симметрии степени $n(n+1) / 2$, т.е. об $n(n+1) / 2$-параметрической группе движений $n$-мерного многообразия (см. $[13,16])$. Ниже даются точные определения, в которых вместо аксиомы феноменологической симметрии приводится аксиома максимальной подвижности.

Классификации двумерных и трехмерных геометрий локальной максимальной подвижности (феноменологически симметричных геометрий), о которых было сказано выше, построены сложными методами, которые неприменимы, ввиду больших технических трудностей, при построении классификаций геометрий размерности 4 и выше. Автором разработан новый метод классификации геометрий локальной максимальной подвижности, названный методом вложения (см. $[6-8,10,11])$. Поясним его суть на хорошо известном примере. Рассмотрим трехмерную геометрию Евклида, задаваемую функцией пары точек:

$$
g(A, B)=\left(x_{A}-x_{B}\right)^{2}+\left(y_{A}-y_{B}\right)^{2}+\left(z_{A}-z_{B}\right)^{2} .
$$

Эта функция является компонентой функций пар точек только следующих четырехмерных геометрий локальной максимальной подвижности:

$$
\begin{aligned}
& f(A, B)=\left(x_{A}-x_{B}\right)^{2}+\left(y_{A}-y_{B}\right)^{2}+\left(z_{A}-z_{B}\right)^{2}+\left(w_{A}-w_{B}\right)^{2}, \\
& f(A, B)=\left(x_{A}-x_{B}\right)^{2}+\left(y_{A}-y_{B}\right)^{2}+\left(z_{A}-z_{B}\right)^{2}-\left(w_{A}-w_{B}\right)^{2}, \\
& f(A, B)=\frac{\left(x_{A}-x_{B}\right)^{2}+\left(y_{A}-y_{B}\right)^{2}+\left(z_{A}-z_{B}\right)^{2}+\left(w_{A}-w_{B}\right)^{2}}{4 w_{A} w_{B}}
\end{aligned}
$$




$$
f(A, B)=\frac{\left(x_{A}-x_{B}\right)^{2}+\left(y_{A}-y_{B}\right)^{2}+\left(z_{A}-z_{B}\right)^{2}-\left(w_{A}-w_{B}\right)^{2}}{4 w_{A} w_{B}} .
$$

Таким образом, $n$-мерная геометрия локальной максимальной подвижности с функцией пары точек $g$ вложена в $(n+1)$-мерную геометрию локальной максимальной подвижности, если ее функция пары точек представима в виде:

$$
f(A, B)=\chi\left(g\left(x_{A}, y_{A}, z_{A}, x_{B}, y_{B}, z_{B}\right), w_{A}, w_{B}\right) .
$$

Метод вложения сводится к решению специальных функциональных уравнений.

2. Точные определения. Рассмотрим $(s+1)$-мерное аналитическое многообразие $M_{s+1}, s=$ 2,3 , которое локально диффеоморфно прямому произведению $s$-мерного аналитического многообразия $N_{s}$ и одномерного аналитического многообразия $L$. Локальный диффеоморфизм осуществляет аналитическое отображение $h_{s}: M_{s+1} \rightarrow N_{s} \times L$. Пусть

$$
\pi_{1}: N_{s} \times L \rightarrow N_{s}, \quad \pi_{2}: N_{s} \times L \rightarrow L
$$

- проекции. Рассмотрим функции $g_{s}: N_{s} \times N_{s} \rightarrow \mathbb{R}$ с открытой и плотной областью определения $S_{g}$ в $N_{s}^{2}$, и $\chi: \mathbb{R} \times L \times L \rightarrow \mathbb{R}$. Определим проекции

$$
p_{1}: M_{s+1} \times M_{s+1} \rightarrow M_{s+1}, \quad p_{2}: M_{s+1} \times M_{s+1} \rightarrow M_{s+1},
$$

которые на точках действуют следующим образом:

$$
p_{1}:\langle A, B\rangle \mapsto A, \quad p_{2}:\langle A, B\rangle \mapsto B,
$$

где $\langle A, B\rangle$ - произвольная точка в $M_{s+1} \times M_{s+1}$. Построим функцию $f: M_{s+1} \times M_{s+1} \rightarrow \mathbb{R}$ по следующей формуле:

$$
f=\chi\left(g_{s}\left(\pi_{1}\left(h_{s}\left(p_{1}\right)\right), \pi_{1}\left(h_{s}\left(p_{2}\right)\right)\right), \pi_{2}\left(h_{s}\left(p_{1}\right)\right), \pi_{2}\left(h_{s}\left(p_{2}\right)\right)\right),
$$

область определения $S_{f}$ которой открыта и плотна в $M_{s+1}^{2}$. Действие на точках имеет вид

$$
\begin{aligned}
f(A, B)=\chi\left(g _ { s } \left(\pi_{1}\left(h_{s}\left(p_{1}(\langle A, B\rangle)\right)\right),\right.\right. & \left.\pi_{1}\left(h_{s}\left(p_{2}(\langle A, B\rangle)\right)\right)\right), \\
& \pi_{2}\left(h_{s}\left(p_{1}(\langle A, B\rangle)\right)\right), \pi_{2}\left(h_{s}\left(p_{2}(\langle A, B\rangle)\right)\right),
\end{aligned}
$$

где $A, B$ - две произвольные точки из $M_{s+1}$, причем $\langle A, B\rangle \in S_{f}$.

Для произвольной точки из $M_{s+1}$ рассмотрим координатную окрестность $U_{s+1} \subset M_{s+1}$, в которой $h_{s}$ является диффеоморфизмом, и для любых точек $A, B \in U_{s+1},\langle A, B\rangle \in S_{f}$, существуют такие окрестности $U_{s+1}(A) \subset U_{s+1}, U_{s+1}(B) \subset U_{s+1}$, что $\left\langle A^{\prime}, B^{\prime}\right\rangle \in S_{f}$ для любых $A^{\prime} \in U_{s+1}(A)$ и $B^{\prime} \in U_{s+1}(B)$. Из сказанного выше следует диффеоморфизм окрестностей $h_{s}: U_{s+1} \rightarrow V_{s} \times W$, где $V_{s}, W$ - некоторые координатные окрестности в $N_{s}$ и $L$ соответственно. Координаты в окрестности $V_{2}$ обозначим $(x, y)$, в окрестности $V_{3}-(x, y, z)$, а в окрестности $W-(w)$. Тогда в локальных координатах функция (1) принимает следующий вид:

$$
f=f(A, B)=\chi\left(\theta, w_{A}, w_{B}\right),
$$

где для двумерного случая $g_{2}\left(\pi_{1}(h(A)), \pi_{1}(h(B))\right)=\theta=\theta\left(x_{A}, y_{A}, x_{B}, y_{B}\right)$ - функция пары точек двумерной геометрии локальной максимальной подвижности, а для трехмерного случая $g_{3}\left(\pi_{1}(h(A)), \pi_{1}(h(B))\right)=\theta=\theta\left(x_{A}, y_{A}, z_{A}, x_{B}, y_{B}, z_{B}\right)$ - функция пары точек трехмерной геометрии локальной максимальной подвижности, $\pi_{2}(h(A))=w_{A}, \pi_{2}(h(B))=w_{B}$. Пусть выполняются следующие аксиомы.

Ажсиома аналитичности: функция $\chi: \mathbb{R} \times L \times L \rightarrow \mathbb{R}$ аналитическая во всех точках области определения;

Ажсиома невырожденности: для функции (2) в произвольной точке окрестности $U_{s+1}(A) \times$ $U_{s+1}(B) \subset M_{s+1}^{2}$ справедливы неравенства

$$
\frac{\partial \chi}{\partial \theta} \neq 0, \quad \frac{\partial \chi}{\partial w_{A}} \neq 0, \quad \frac{\partial \chi}{\partial w_{B}} \neq 0
$$


Пусть группа Ли $G$ действует эффективно и аналитично в $U_{s+1} \subset M_{s+1}$ (см. $\left.[2,13]\right)$. Это означает, что задано аналитическое инъективное отображение (эффективное действие) $\lambda: U_{s+1} \times$ $G \rightarrow U_{s+1}^{\prime}$, где $U_{s+1}^{\prime} \subset M_{s+1}$ - открытая область, причем выполняются следующие свойства:

(i) $\lambda(A, e)=A, e \in G$ - единица, $A \in U_{s+1}$;

(ii) $\lambda(\lambda(A, a), b)=\lambda(A, a b)$, для любых $a, b \in G$ и $A \in U_{s+1}$;

(iii) Для любого $A \in U_{s+1} \lambda(A, a)=A$, только если $a=e$.

Действие $\lambda_{a}$, определяемое произвольным элементом $a \in G$, называется движением, если для любых точек $A, B \in U_{s+1}$ таких, что $\langle A, B\rangle \in S_{f},\left\langle\lambda_{a}(A), \lambda_{a}(B)\right\rangle \in S_{f}$, выполняется равенство

$$
f\left(\lambda_{a}(A), \lambda_{a}(B)\right)=f(A, B) .
$$

Действия группы $G$ можно определить в окрестностях $U_{s+1}(A)$ и $U_{s+1}(B)$ точек $A$ и $B$, причем если эти окрестности пересекаются, то действия в пересечении совпадают. Множество всех движений образует группу движений.

Аксиома максимальной подвижности: размерность группы Ли $G$ равна $(s+1)(s+2) / 2$.

Алгебра Ли действия группы Ли $G$ при $s=2$ состоит из операторов

$$
X=X_{1} \partial_{x}+X_{2} \partial_{y}+P \partial_{w},
$$

где $X_{\alpha}=X_{\alpha}(x, y, w), P=P(x, y, w)$ - аналитические функция в $U_{3}, \alpha=1,2$ (см. [13]), а при $s=3$ - из операторов

$$
X=X_{1} \partial_{x}+X_{2} \partial_{y}+X_{3} \partial_{z}+P \partial_{w}
$$

$X_{\beta}=X_{\beta}(x, y, z, w), P=P(x, y, z, w)$ - аналитические функция в $U_{4}, \beta=1,2,3$ (см. [13]).

Через операторы (4) и (5) записывается условие локальной инвариантности (см. $[13,14])$

$$
X(A) f(A, B)+X(B) f(A, B)=0,
$$

которое выполняется в окрестностях $U_{s+1}(A) \subset U_{s+1}$ и $U(B)_{s+1} \subset U_{s+1}$ точек $A$ и $B$.

Можно найти все функции пары точек вида (2), являющиеся двухточечными инвариантами группы движений размерности $(s+1)(s+2) / 2=6(s=2)$, причем $\theta$ - функция пары точек, задающая двумерную геометрию локальной максимальной подвижности. Список таких геометрий приводится ниже. Аналогично можно найти все функции пары точек вида (2), являющиеся двухточечными инвариантами группы движений размерности $(s+1)(s+2) / 2=10(s=3)$, причем $\theta-$ функция пары точек, задающая трехмерную геометрию локальной максимальной подвижности.

Эта задача аналитически решается в $[6,7,10,11]$. Для ее решения компоненты операторов (4) и (5) алгебр Ли групп движений, а также функции пары точек представляются рядами Тейлора и подставляются в функциональные уравнения специального вида, которые получаются из условия локальной инвариантности (2) при подстановке в них компонент операторов (4), (5) и функции (2). Пусть $k_{s} \in U_{s+1} \subset M_{s+1}$ - начало некоторой системы координат в $U_{s+1}$, в которой эта точка имеет нулевые координаты. В такой системе координат справедливы разложения в ряд Тейлора для компонент операторов (4), (5) и функции (2) (см. [15]):

$$
\begin{aligned}
X_{1}=X_{1}(w)+D_{1}\left(X_{1}\right)(w) x+D_{2}\left(X_{1}\right)(w) y+ & \frac{1}{2} D_{1,1}\left(X_{1}\right)(w) x^{2}+ \\
& +\frac{1}{2} D_{2,2}\left(X_{1}\right)(w) y^{2}+D_{1,2}\left(X_{1}\right)(w) x y+\ldots \\
X_{2}=X_{2}(w)+D_{1}\left(X_{2}\right)(w) x+D_{2}\left(X_{2}\right)(w) y+\frac{1}{2} D_{1,1}\left(X_{2}\right)(w) x^{2}+ & \\
& +\frac{1}{2} D_{2,2}\left(X_{2}\right)(w) y^{2}+D_{1,2}\left(X_{2}\right)(w) x y+\ldots
\end{aligned}
$$

$$
\begin{aligned}
P=P(w)+D_{1}(P)(w) x+D_{2}(P)(w) y+\frac{1}{2} D_{1,1}(P)(w) & x^{2}+ \\
& +\frac{1}{2} D_{2,2}(P)(w) y^{2}+D_{1,2}(P)(w) x y+\ldots
\end{aligned}
$$




$$
\begin{aligned}
& X_{1}=X_{1}(z, w)+D_{1}\left(X_{1}\right)(z, w) x+D_{2}\left(X_{1}\right)(z, w) y+\frac{1}{2} D_{1,1}\left(X_{1}\right)(z, w) x^{2}+ \\
&+ \frac{1}{2} D_{2,2}\left(X_{1}\right)(z, w) y^{2}+D_{1,2}\left(X_{1}\right)(z, w) x y+\ldots, \\
& X_{2}=X_{2}(z, w)+D_{1}\left(X_{2}\right)(z, w) x+D_{2}\left(X_{2}\right)(z, w) y+\frac{1}{2} D_{1,1}\left(X_{2}\right)(z, w) x^{2}+ \\
&+ \frac{1}{2} D_{2,2}\left(X_{2}\right)(z, w) y^{2}+D_{1,2}\left(X_{2}\right)(z, w) x y+\ldots \\
& X_{3}=X_{3}(z, w)+D_{1}\left(X_{3}\right)(z, w) x+D_{2}\left(X_{3}\right)(z, w) y+\frac{1}{2} D_{1,1}\left(X_{3}\right)(z, w) x^{2}+ \\
&+\frac{1}{2} D_{2,2}\left(X_{3}\right)(z, w) y^{2}+D_{1,2}\left(X_{3}\right)(z, w) x y+\ldots \\
& P=P(z, w)+D_{1}(P)(z, w) x+D_{2}(P)(z, w) y+ \frac{1}{2} D_{1,1}(P)(z, w) x^{2}+ \\
&+\frac{1}{2} D_{2,2}(P)(z, w) y^{2}+D_{1,2}(P)(z, w) x y+\ldots \\
& f\left(\theta, w_{A}, w_{B}\right)=f\left(w_{A}, w_{B}\right)+D_{1}(f)\left(w_{A}, w_{B}\right) \theta+\frac{1}{2} D_{1,1}(f)\left(w_{A}, w_{B}\right) \theta^{2}+\ldots
\end{aligned}
$$

где, например,

$$
\begin{gathered}
X_{1}(w)=X_{1}(0,0, w), \quad P(z, w)=P(0,0, z, w), \\
D_{1}\left(X_{1}\right)(w)=\left.\frac{\partial X_{1}(x, y, w)}{\partial x}\right|_{x=y=0}, \quad D_{2}\left(X_{1}\right)(z, w)=\left.\frac{\partial X_{1}(x, y, z, w)}{\partial y}\right|_{x=y=0}, \\
D_{1,2}\left(X_{2}\right)(w)=\left.\frac{\partial^{2} X_{2}(x, y, w)}{\partial x \partial y}\right|_{x=y=0}, \quad D_{1,1}(P)(z, w)=\left.\frac{\partial^{2} P(x, y, z, w)}{\partial x \partial x}\right|_{x=y=0}, \\
f\left(w_{A}, w_{B}\right)=\chi\left(0, w_{A}, w_{B}\right), \quad D_{1}(f)\left(w_{A}, w_{B}\right)=\left.\frac{\partial \chi\left(\theta, w_{A}, w_{B}\right)}{\partial \theta}\right|_{\theta=0} .
\end{gathered}
$$

3. Двумерные, трехмерные и четырехмерные геометрии локальной максимальной подвижности. В данном параграфе приводятся функции пары точек двумерных, трехмерных и четырехмерных геометрий локальной максимальной подвижности.

3.1. Двумерные геометрии локальной максимальной подвиљности. Известен полный список двумерных геометрий локальной максимальной подвижности, содержащей геометрии с функциями пары точек:

$$
\begin{aligned}
& f(A, B)=\sin y_{A} \sin y_{B} \cos \left(x_{A}-x_{B}\right)+\cos y_{A} \cos y_{B} \\
& f(A, B)=\operatorname{sh} y_{A} \operatorname{sh} y_{B} \cos \left(x_{A}-x_{B}\right)-\operatorname{ch} y_{A} \operatorname{ch} y_{B} \\
& f(A, B)=\operatorname{sh} y_{A} \operatorname{sh} y_{B} \operatorname{ch}\left(x_{A}-x_{B}\right)-\operatorname{ch} y_{A} \operatorname{ch} y_{B} \\
& f(A, B)=\left(x_{A}-x_{B}\right)^{2}+\left(y_{A}-y_{B}\right)^{2} \\
& f(A, B)=\left(x_{A}-x_{B}\right)^{2}-\left(y_{A}-y_{B}\right)^{2} \\
& f(A, B)=x_{A} y_{B}-x_{B} y_{A} \\
& f(A, B)=\frac{y_{A}-y_{B}}{x_{A}-x_{B}} \\
& f(A, B)=\left(\left(x_{A}-x_{B}\right)^{2}+\left(y_{A}-y_{B}\right)^{2}\right) e^{2 \gamma \operatorname{arctg} \frac{y_{A}-y_{B}}{x_{A}-x_{B}}} \\
& f(A, B)=\beta \ln \left(y_{A}-y_{B}\right)+\varepsilon \ln \left(x_{A}-X_{B}\right)
\end{aligned}
$$




$$
f(A, B)=\left(x_{A}-x_{B}\right)^{2} e^{2 \frac{y_{A}-y_{B}}{x_{A}-x_{B}}},
$$

где $\beta, \gamma=$ const, $\gamma \neq 0, \beta \neq 0, \pm 1, \varepsilon= \pm 1$. Эти геометрии получены Г. Г. Михайличенко при классификации двумерных феноменологически симметричных геометрий (см. $[13,16])$.

Функция (10) задает сферическую геометрию в проективной интерпретации, т.е. на сфере в трехмерном евклидовом пространстве, задаваемой уравнением $\alpha_{1}^{2}+\alpha_{2}^{2}+\alpha_{3}^{2}=1$. Можно перейти к другой функции пары точек: $l(A, B)=(1-f(A, B)) / 2$, которая в новых координатах $u=x, v=\operatorname{ctg} \frac{y}{2}$ (стереографическая проекция) принимает вид (см. [4]):

$$
l(A, B)=\frac{v_{A}^{2}+v_{B}^{2}-2 v_{A} v_{B} \cos \left(u_{A}-u_{B}\right)}{\left(v_{A}^{2}+1\right)\left(v_{B}^{2}+1\right)} .
$$

Введем еще одну замену координат: $p=v \cos u, q=v \sin u$, тогда функция $l(A, B)$ примет новый вид:

$$
l(A, B)=\frac{\left(p_{A}-p_{B}\right)^{2}+\left(q_{A}-q_{B}\right)^{2}}{\left(p_{A}^{2}+q_{A}^{2}+1\right)\left(p_{B}^{2}+q_{B}^{2}+1\right)} .
$$

Функция (11) задает геометрию Лобачевского в проективной интерпретации, т.е. на двуполостном гиперболоиде в трехмерном псевдоевклидовом пространстве сигнатуры $(+,+,-)$, задаваемом уравнением $\alpha_{1}^{2}+\alpha_{2}^{2}-\alpha_{3}^{2}=-1$. Можно также перейти к функции: $l(A, B)=(1+f(A, B)) / 2$, которая в координатах $u=x, v=\operatorname{cth} \frac{y}{2}$ (стереографическая проекция) принимает вид (см. [4]):

$$
l(A, B)=\frac{v_{A}^{2}+v_{B}^{2}-2 v_{A} v_{B} \cos \left(u_{A}-u_{B}\right)}{\left(v_{A}^{2}-1\right)\left(v_{B}^{2}-1\right)} .
$$

Введем, как и выше, замену координат: $p=v \cos u, q=v \sin u$, тогда для $l(A, B)$ получаем:

$$
l(A, B)=\frac{\left(p_{A}-p_{B}\right)^{2}+\left(q_{A}-q_{B}\right)^{2}}{\left(p_{A}^{2}+q_{A}^{2}-1\right)\left(p_{B}^{2}+q_{B}^{2}-1\right)} .
$$

Функция (12) задает двумерную геометрию де Ситтера в проективной интерпретации, т.е. на однополосном гиперболоиде в трехмерном псевдоевклидовом пространстве сигнатуры $(+,+,-)$, задаваемом уравнением $\alpha_{1}^{2}+\alpha_{2}^{2}-\alpha_{3}^{2}=1$. Можно перейти к функции: $l(A, B)=(1+f(A, B)) / 2$, которая в координатах $u=x, v=\operatorname{cth} \frac{y}{2}$ (стереографическая проекция) принимает вид:

$$
l(A, B)=\frac{-v_{A}^{2}-v_{B}^{2}+2 v_{A} v_{B} \operatorname{ch}\left(u_{A}-u_{B}\right)}{\left(v_{A}^{2}-1\right)\left(v_{B}^{2}-1\right)} .
$$

Введем замену координат: $p=v \operatorname{sh} u, q=v \operatorname{ch} u$, тогда $l(A, B)$ получаем:

$$
l(A, B)=\frac{\left(p_{A}-p_{B}\right)^{2}-\left(q_{A}-q_{B}\right)^{2}}{\left(p_{A}^{2}-q_{A}^{2}-1\right)\left(p_{B}^{2}-q_{B}^{2}-1\right)} .
$$

Функция (13) задает евклидову геометрию, функция (14) - псевдоевклидову геометрию, (15) симплектическую геометрию, (16) - симплициальную геометрию, (17) - собственно гельмгольцеву геометрию, (18) - псевдогельмгольцеву геометрию и, наконец, (19) - дуальногельмгольцеву геометрию.

Функцию (20) легко записать в комплексной форме:

$$
l(A, B)=\frac{\left(z_{A}-z_{B}\right) \overline{\left(z_{A}-z_{B}\right)}}{\left(z_{A} \overline{z_{A}}+1\right)\left(z_{B} \overline{z_{B}}+1\right)}
$$

где $z=p+i q, i^{2}=-1$. Функцию (21) также легко записать в комплексной форме:

$$
l(A, B)=\frac{\left(z_{A}-z_{B}\right) \overline{\left(z_{A}-z_{B}\right)}}{\left(z_{A} \overline{z_{A}}-1\right)\left(z_{B} \overline{z_{B}}-1\right)} .
$$


Рассмотрим дробно-линейное преобразование $z=\frac{1+i w}{1-i w}$, где $w=x+i y$, которое функцию $(24)$ приводит к виду (см. [4])

$$
l(A, B)=-\frac{\left(w_{A}-w_{B}\right) \overline{\left(w_{A}-w_{B}\right)}}{\left(w_{A}-\overline{w_{A}}\right)\left(w_{B}-\overline{w_{B}}\right)} .
$$

Переходя к декартовым координатам, получаем

$$
l(A, B)=\frac{\left(x_{A}-x_{B}\right)^{2}+\left(y_{A}-y_{B}\right)^{2}}{4 y_{A} y_{B}} .
$$

Функцию (22) можно записать, используя двойные числа:

$$
l(A, B)=\frac{\left(z_{A}-z_{B}\right) \overline{\left(z_{A}-z_{B}\right)}}{\left(z_{A} \overline{z_{A}}+1\right)\left(z_{B} \overline{z_{B}}+1\right)},
$$

где $z=p+i q, \bar{z}=p-i q,|z|^{2}=z \bar{z}=p^{2}-q^{2}, i^{2}=1$. Рассмотрим дробно-линейное преобразование $z=i \frac{1+i w}{1-i w}$, где $w=x+i y$, которое функцию (26) приводит к виду

$$
l(A, B)=\frac{\left(w_{A}-w_{B}\right) \overline{\left(w_{A}-w_{B}\right)}}{\left(w_{A}-\overline{w_{A}}\right)\left(w_{B}-\overline{w_{B}}\right)} .
$$

Переходя к декартовым координатам, получаем

$$
f(A, B)=\frac{\left(x_{A}-x_{B}\right)^{2}-\left(y_{A}-y_{B}\right)^{2}}{4 y_{A} y_{B}} .
$$

От функций пары точек (10)-(12) удобно перейти к следующим функциям:

$$
\begin{aligned}
& f(A, B)=m_{A} m_{B}+n_{A} n_{B}+l_{A} l_{B}, m^{2}+n^{2}+l^{2}>0 ; \\
& f(A, B)=m_{A} m_{B}+n_{A} n_{B}-l_{A} l_{B}, m^{2}+n^{2}-l^{2}<0 ; \\
& f(A, B)=m_{A} m_{B}-n_{A} n_{B}-l_{A} l_{B}, m^{2}-n^{2}-l^{2}<0 .
\end{aligned}
$$

Заметим, что на сфере функции пары точек (10) и (28) совпадают; группы движений, сохраняющие эти функции также совпадают. Подобный вывод справедлив и для функция пар точек (11) и (29), а также для функций (12) и (30).

3.2. Трехмерные геометрии локальной максимальной подвиюннсти. Известен список трехмерных геометрий локальной максимальной подвижности, содержащей геометрии, задаваемые функциями пары точек:

$$
\begin{aligned}
& f(A, B)=\sin z_{A} \sin z_{B}\left[\sin y_{A} \sin y_{B} \cos \left(x_{A}-x_{B}\right)+\cos y_{A} \cos y_{B}\right]+\cos z_{A} \cos z_{B} \\
& f(A, B)=\operatorname{ch} z_{A} \operatorname{ch} z_{B}\left[\operatorname{sh} y_{A} \operatorname{sh} y_{B} \cos \left(x_{A}-x_{B}\right)-\operatorname{ch} y_{A} \operatorname{ch} y_{B}\right]+\operatorname{sh} z_{A} \operatorname{sh} z_{B} \\
& f(A, B)=\operatorname{sh} z_{A} \operatorname{sh} z_{B}\left[\operatorname{sh} y_{A} \operatorname{sh} y_{B} \cos \left(x_{A}-x_{B}\right)-\operatorname{ch} y_{A} \operatorname{ch} y_{B}\right]+\operatorname{ch} z_{A} \operatorname{ch} z_{B} \\
& f(A, B)=\operatorname{sh} z_{A} \operatorname{sh} z_{B}\left[\operatorname{sh} y_{A} \operatorname{sh} y_{B} \operatorname{ch}\left(x_{A}-x_{B}\right)-\operatorname{ch} y_{A} \operatorname{ch} y_{B}\right]+\operatorname{ch} z_{A} \operatorname{ch} z_{B} \\
& f(A, B)=\left(x_{A}-x_{B}\right)^{2}+\left(y_{A}-y_{B}\right)^{2}+\left(z_{A}-z_{B}\right)^{2} \\
& f(A, B)=\left(x_{A}-x_{B}\right)^{2}+\left(y_{A}-y_{B}\right)^{2}-\left(z_{A}-z_{B}\right)^{2} \\
& f(A, B)=x_{A} y_{B}-x_{B} y_{A}+z_{A}-z_{B} \\
& f(A, B)=\frac{y_{A}-y_{B}}{x_{A}-x_{B}}+z_{A}+z_{B} \\
& f(A, B)=\frac{y_{A}-y_{B}}{x_{A}-x_{B}} e^{z_{A}+z_{B}} \\
& f(A, B)=\operatorname{arctg} \frac{y_{A}-y_{B}}{x_{A}-x_{B}}+z_{A}+z_{B} \\
& f(A, B)=\left(\left(x_{A}-x_{B}\right)^{2}+\left(y_{A}-y_{B}\right)^{2}\right) e^{2 z_{A}+2 z_{B}} \\
& f(A, B)=\left(\left(x_{A}-x_{B}\right)^{2}-\left(y_{A}-y_{B}\right)^{2}\right) e^{2 z_{A}+2 z_{B}}
\end{aligned}
$$




$$
\begin{aligned}
& f(A, B)=\left(\left(x_{A}-x_{B}\right)^{2}+\left(y_{A}-y_{B}\right)^{2}\right) e^{2 \gamma \operatorname{arctg} \frac{y_{A}-y_{B}}{x_{A}-x_{B}}+2 z_{A}+2 z_{B}} ; \\
& f(A, B)=\beta \ln \left(y_{A}-y_{B}\right)+\varepsilon \ln \left(x_{A}-X_{B}\right)+z_{A}+z_{B} ; \\
& f(A, B)=\left(x_{A}-x_{B}\right)^{2} e^{2 \frac{y_{A}-y_{B}}{x_{A}-x_{B}}+2 z_{A}+2 z_{B}},
\end{aligned}
$$

где $\beta, \gamma=$ const, $\gamma \neq 0, \beta \neq 0, \pm 1, \varepsilon= \pm 1$. Геометрии из этого списка, кроме (40), получены В. Х. Левом при классификации трехмерных феноменологически симметричных геометрий (см. [12]). Геометрия (40) найдена В. А. Кыровым (см. [9]). Функция (31) задает сферическую геометрию в проективной интерпретации, т.е. геометрию на сфере в четырехмерном евклидовом пространстве, задаваемой уравнением $\alpha_{1}^{2}+\alpha_{2}^{2}+\alpha_{3}^{2}+\alpha_{4}^{2}=1$. Перейдем к другой функции $l(A, B)=(1-f(A, B)) / 2$ и введем новые координаты с помощью стереографической проекции (см. [3]):

$$
\begin{aligned}
\sin z \sin y \cos x & =\frac{2 p}{p^{2}+q^{2}+r^{2}+1}, & \sin z \sin y \sin x & =\frac{2 q}{p^{2}+q^{2}+r^{2}+1} \\
\sin z \cos y & =\frac{2 r}{p^{2}+q^{2}+r^{2}+1}, & \cos z & =\frac{p^{2}+q^{2}+r^{2}-1}{p^{2}+q^{2}+r^{2}+1}
\end{aligned}
$$

Тогда

$$
l(A, B)=\frac{\left(p_{A}-p_{B}\right)^{2}+\left(q_{A}-q_{B}\right)^{2}+\left(r_{A}-r_{B}\right)^{2}}{\left(p_{A}^{2}+q_{A}^{2}+r_{A}^{2}+1\right)\left(p_{B}^{2}+q_{B}^{2}+r_{B}^{2}+1\right)} .
$$

Функция (32) задает геометрию Лобачевского в проективной интерпретации, т.е. геометрию на двуполостном гиперболоиде в четырехмерном псевдоевклидовом пространстве сигнатуры $(+,+,+,-)$, задаваемом уравнением $\alpha_{1}^{2}+\alpha_{2}^{2}+\alpha_{3}^{2}-\alpha_{4}^{2}=-1$. Рассмотрим другую функцию пары точек: $l(A, B)=-(1+f(A, B)) / 2$. Введем стереографические координаты по формулам

$$
\begin{aligned}
\operatorname{ch} z \operatorname{sh} y \cos x & =\frac{2 p}{p^{2}+q^{2}+r^{2}-1}, & \operatorname{ch} z \operatorname{sh} y \sin x & =\frac{2 q}{p^{2}+q^{2}+r^{2}-1}, \\
\operatorname{ch} z \operatorname{ch} y & =\frac{p^{2}+q^{2}+r^{2}+1}{p^{2}+q^{2}+r^{2}-1}, & \operatorname{sh} z & =\frac{2 r}{p^{2}+q^{2}+r^{2}-1} .
\end{aligned}
$$

Тогда

$$
l(A, B)=\frac{\left(p_{A}-p_{B}\right)^{2}+\left(q_{A}-q_{B}\right)^{2}+\left(r_{A}-r_{B}\right)^{2}}{\left(p_{A}^{2}+q_{A}^{2}+r_{A}^{2}-1\right)\left(p_{B}^{2}+q_{B}^{2}+r_{B}^{2}-1\right)} .
$$

Функция (33) задает геометрию де Ситтера в проективной интерпретации, т.е. геометрию на однополостном гиперболоиде в четырехмерном псевдоевклидовом пространстве сигнатуры $(+,+,+,-)$, задаваемом уравнением $\alpha_{1}^{2}+\alpha_{2}^{2}+\alpha_{3}^{2}-\alpha_{4}^{2}=1$. Переходим к другой функции $l(A, B)=(1-f(A, B)) / 2$. Введем стереографические координаты

$$
\begin{aligned}
\operatorname{sh} z \operatorname{sh} y \cos x & =\frac{2 p}{p^{2}+q^{2}-r^{2}+1}, & \operatorname{sh} z \operatorname{sh} y \sin x & =\frac{2 q}{p^{2}+q^{2}-r^{2}+1}, \\
\operatorname{sh} z \operatorname{ch} y & =\frac{2 r}{p^{2}+q^{2}-r^{2}+1}, & \operatorname{ch} z & =\frac{p^{2}+q^{2}-r^{2}-1}{p^{2}+q^{2}-r^{2}+1} .
\end{aligned}
$$

Тогда

$$
l(A, B)=\frac{\left(p_{A}-p_{B}\right)^{2}+\left(q_{A}-q_{B}\right)^{2}-\left(r_{A}-r_{B}\right)^{2}}{\left(p_{A}^{2}+q_{A}^{2}-r_{A}^{2}+1\right)\left(p_{B}^{2}+q_{B}^{2}-r_{B}^{2}+1\right)} .
$$

Функция (34) задает антидеситтерову геометрию в проективной интерпретации, т.е. геометрию на гиперболоиде в четырехмерном псевдоевклидовом пространстве сигнатуры $(+,+,-,-)$, задаваемом уравнением $\alpha_{1}^{2}+\alpha_{2}^{2}-\alpha_{3}^{2}-\alpha_{4}^{2}=-1$. Переходим к другой функции пары точек $l(A, B)=(1+f(A, B)) / 2$. В стереографических координатах будем иметь

$$
\begin{aligned}
\operatorname{sh} z \operatorname{sh} y \operatorname{ch} x & =\frac{2 p}{p^{2}+q^{2}-r^{2}-1}, & \operatorname{sh} z \operatorname{sh} y \operatorname{sh} x & =\frac{p^{2}+q^{2}-r^{2}+1}{p^{2}+q^{2}-r^{2}-1}, \\
\operatorname{sh} z \operatorname{ch} y & =\frac{2 r}{p^{2}+q^{2}-r^{2}-1}, & \operatorname{ch} z & =\frac{2 q}{p^{2}+q^{2}-r^{2}-1} .
\end{aligned}
$$


Тогда

$$
l(A, B)=-\frac{\left(p_{A}-p_{B}\right)^{2}+\left(q_{A}-q_{B}\right)^{2}-\left(r_{A}-r_{B}\right)^{2}}{\left(p_{A}^{2}+q_{A}^{2}-r_{A}^{2}-1\right)\left(p_{B}^{2}+q_{B}^{2}-r_{B}^{2}-1\right)} .
$$

Функция (35) задает евклидову геометрию, функция (36) - псевдоевклидову геометрию, функция (37) - симплектическую трехмерную геометрию, функция (38) - симплициальную геометрию I типа, функция (39) - симплициальную геометрию II типа, функция (40) - симплициальную геометрию III типа, функция (41) - особое расширение евклидовой геометрии, функция (42) особое расширение псевдоевклидовой геометрии, функция (43) - собственно гельмгольцеву геометрию, функция (44) - псевдогельмгольцеву геометрию и, наконец, функция (45) - дуальногельмгольцеву геометрию.

В функции пары точек (47) переходим к новым координатам:

$$
p=\frac{2 x}{(1+z)^{2}+x^{2}+y^{2}}, \quad q=\frac{2 y}{(1+z)^{2}+x^{2}+y^{2}}, \quad r=\frac{1-x^{2}-y^{2}-z^{2}}{(1+z)^{2}+x^{2}+y^{2}}
$$

при этом

$$
p^{2}+q^{2}+r^{2}-1=\frac{-4 z}{(1+z)^{2}+x^{2}+y^{2}} .
$$

Тогда функция (47) примет вид

$$
l(A, B)=\frac{\left(x_{A}-x_{B}\right)^{2}+\left(y_{A}-y_{B}\right)^{2}+\left(z_{A}-z_{B}\right)^{2}}{4 z_{A} z_{B}} .
$$

Аналогично, в функции (48) переходим к новым координатам

при этом

$$
p=\frac{2 x}{(1+z)^{2}-x^{2}-y^{2}}, \quad q=\frac{2 y}{(1+z)^{2}-x^{2}-y^{2}}, \quad r=\frac{1+x^{2}+y^{2}-z^{2}}{(1+z)^{2}-x^{2}-y^{2}} ;
$$

$$
p^{2}+q^{2}-r^{2}+1=\frac{4 z}{(1+z)^{2}-x^{2}-y^{2}} .
$$

Тогда (48) примет вид

$$
l(A, B)=\frac{\left(x_{A}-x_{B}\right)^{2}+\left(y_{A}-y_{B}\right)^{2}-\left(z_{A}-z_{B}\right)^{2}}{4 z_{A} z_{B}},
$$

Аналогично, в функции (49) переходим к координатам

при этом

$$
p=\frac{2 y}{(1-z)^{2}-x^{2}+y^{2}}, \quad r=\frac{2 x}{(1-z)^{2}-x^{2}+y^{2}}, \quad q=\frac{1+x^{2}-y^{2}-z^{2}}{(1-z)^{2}-x^{2}+y^{2}}
$$

Тогда (49) примет вид

$$
p^{2}+q^{2}-r^{2}-1=\frac{4 z}{(1-z)^{2}-x^{2}+y^{2}} .
$$

$$
l(A, B)=\frac{\left(x_{A}-x_{B}\right)^{2}-\left(y_{A}-y_{B}\right)^{2}-\left(z_{A}-z_{B}\right)^{2}}{4 z_{A} z_{B}} .
$$

От функций пары точек (31)-(34) удобно перейти к следующим функциям:

$$
\begin{aligned}
& f(A, B)=m_{A} m_{B}+n_{A} n_{B}+l_{A} l_{B}+k_{A} k_{B}, m^{2}+n^{2}+l^{2}+k^{2}>0 ; \\
& f(A, B)=m_{A} m_{B}+n_{A} n_{B}-l_{A} l_{B}+k_{A} k_{B}, m^{2}+n^{2}-l^{2}+k^{2}<0 ; \\
& f(A, B)=m_{A} m_{B}+n_{A} n_{B}-l_{A} l_{B}+k_{A} k_{B}, m^{2}+n^{2}-l^{2}+k^{2}>0 ; \\
& f(A, B)=m_{A} m_{B}-n_{A} n_{B}-l_{A} l_{B}+k_{A} k_{B}, m^{2}-n^{2}-l^{2}+k^{2}>0 .
\end{aligned}
$$

Заметим, что на сфере функции пары точек (31) и (53) совпадают; группы движений, сохраняющие эти функции также совпадают. Подобный вывод справедлив и для функция пар точек (32) и (54), для функций (33) и (55), а также для функций (34) и (56). 
3.3. Четьрехмерные геометрии локальной максимальной подвижности. Известен список четырехмерных геометрий локальной максимальной подвижности, содержащей геометрии, задаваемые функциями пары точек:

$$
\begin{aligned}
f(A, B)=\sin w_{A} \sin w_{B}\left[\sin z_{A} \sin z_{B} \times\right. \\
\left.\quad \times\left[\sin y_{A} \sin y_{B} \cos \left(x_{A}-x_{B}\right)+\cos y_{A} \cos y_{B}\right]+\cos z_{A} \cos z_{B}\right]+\cos w_{A} \cos w_{B}
\end{aligned}
$$

$f(A, B)=\operatorname{ch} w_{A} \operatorname{ch} w_{B}\left[\operatorname{ch} z_{A} \operatorname{ch} z_{B} \times\right.$

$$
\left.\times\left[\operatorname{sh} y_{A} \operatorname{sh} y_{B} \cos \left(x_{A}-x_{B}\right)-\operatorname{ch} y_{A} \operatorname{ch} y_{B}\right]+\operatorname{sh} z_{A} \operatorname{sh} z_{B}\right]+\operatorname{sh} w_{A} \operatorname{sh} w_{B}
$$

$$
\begin{aligned}
f(A, B)=\operatorname{sh} w_{A} \operatorname{sh} w_{B} & {\left[\operatorname{ch} z_{A} \operatorname{ch} z_{B} \times\right.} \\
\times & {\left.\left[\operatorname{sh} y_{A} \operatorname{sh} y_{B} \cos \left(x_{A}-x_{B}\right)-\operatorname{ch} y_{A} \operatorname{ch} y_{B}\right]+\operatorname{sh} z_{A} \operatorname{sh} z_{B}\right]+\operatorname{ch} w_{A} \operatorname{ch} w_{B} }
\end{aligned}
$$

$f(A, B)=\operatorname{ch} w_{A} \operatorname{ch} w_{B}\left[\operatorname{sh} z_{A} \operatorname{sh} z_{B} \times\right.$

$$
\left.\times\left[\operatorname{sh} y_{A} \operatorname{sh} y_{B} \cos \left(x_{A}-x_{B}\right)-\operatorname{ch} y_{A} \operatorname{ch} y_{B}\right]+\operatorname{ch} z_{A} \operatorname{ch} z_{B}\right]-\operatorname{sh} w_{A} \operatorname{sh} w_{B}
$$

$$
\begin{aligned}
& f(A, B)=\operatorname{ch} w_{A} \operatorname{ch} w_{B}\left[\operatorname{sh} z_{A} \operatorname{sh} z_{B} \times\right. \\
&\left.\times\left[\operatorname{sh} y_{A} \operatorname{sh} y_{B} \operatorname{ch}\left(x_{A}-x_{B}\right)-\operatorname{ch} y_{A} \operatorname{ch} y_{B}\right]+\operatorname{ch} z_{A} \operatorname{ch} z_{B}\right]-\operatorname{sh} w_{A} \operatorname{sh} w_{B} \\
& f(A, B)=\left(x_{A}-x_{B}\right)^{2}+\left(y_{A}-y_{B}\right)^{2}+\left(z_{A}-z_{B}\right)^{2}+\left(w_{A}-w_{B}\right)^{2} \\
& f(A, B)=\left(x_{A}-x_{B}\right)^{2}+\left(y_{A}-y_{B}\right)^{2}+\left(z_{A}-z_{B}\right)^{2}-\left(w_{A}-w_{B}\right)^{2} \\
& f(A, B)=\left(x_{A}-x_{B}\right)^{2}+\left(y_{A}-y_{B}\right)^{2}-\left(z_{A}-z_{B}\right)^{2}-\left(w_{A}-w_{B}\right)^{2} \\
& f(A, B)=\left(\left(x_{A}-x_{B}\right)^{2}+\left(y_{A}-y_{B}\right)^{2}+\left(z_{A}-z_{B}\right)^{2}\right) e^{w_{A}+w_{B}} \\
& f(A, B)=\left(\left(x_{A}-x_{B}\right)^{2}+\left(y_{A}-y_{B}\right)^{2}-\left(z_{A}-z_{B}\right)^{2}\right) e^{w_{A}+w_{B}} \\
& f(A, B)=x_{A} y_{B}-x_{B} y_{A}+z_{A} w_{B}-z_{B} w_{A}
\end{aligned}
$$

где $\beta, \gamma=$ const, $\gamma \neq 0, \beta \neq 0, \pm 1$. Геометрии из этого списка найдены В. А. Кыровым (см. [6-8,11]) при классификации феноменологически симметричных геометрий методом вложения. Функция пары точек (57) задает сферическую геометрию в проективной интерпретации, т.е. геометрию на сфере в пятимерном евклидовом пространстве, задаваемой уравнением $\alpha_{1}^{2}+\alpha_{2}^{2}+\alpha_{3}^{2}+\alpha_{4}^{2}+\alpha_{5}^{2}=1$.

Переходим к функции $l(A, B)=(1-f(A, B)) / 2$. Введем стереографические координаты (см. [3]) $\sin w \sin z \sin y \cos x=\frac{2 p}{p^{2}+q^{2}+r^{2}+t^{2}+1}, \quad \sin w \sin z \sin y \sin x=\frac{2 q}{p^{2}+q^{2}+r^{2}+t^{2}+1}$,

$$
\begin{gathered}
\sin w \sin z \cos y=\frac{2 r}{p^{2}+q^{2}+r^{2}+t^{2}+1}, \quad \sin w \cos z=\frac{2 t}{p^{2}+q^{2}+r^{2}+t^{2}+1} \\
\cos w=\frac{p^{2}+q^{2}+r^{2}+t^{2}-1}{p^{2}+q^{2}+r^{2}+t^{2}+1}
\end{gathered}
$$

Тогда

$$
l(A, B)=\frac{\left(p_{A}-p_{B}\right)^{2}+\left(q_{A}-q_{B}\right)^{2}+\left(r_{A}-r_{B}\right)^{2}+\left(t_{A}-t_{B}\right)^{2}}{\left(p_{A}^{2}+q_{A}^{2}+r_{A}^{2}+t_{A}^{2}+1\right)\left(p_{B}^{2}+q_{B}^{2}+r_{B}^{2}+t_{B}^{2}+1\right)} .
$$

Функция (58) задает геометрию Лобачевского в проективной интерпретации, т.е. геометрию на двуполостном гиперболоиде в пятимерном псевдоевклидовом пространстве сигнатуры 
$(+,+,+,+,-)$, задаваемом уравнением $\alpha_{1}^{2}+\alpha_{2}^{2}+\alpha_{3}^{2}+\alpha_{4}^{2}-\alpha_{5}^{2}=-1$. Переходим к функции $l(A, B)=-(1+f(A, B)) / 2$. Введем стереографические координаты:

$$
\begin{gathered}
\operatorname{ch} w \operatorname{ch} z \operatorname{sh} y \cos x=\frac{2 p}{p^{2}+q^{2}+r^{2}+t^{2}-1}, \quad \operatorname{ch} w \operatorname{ch} z \operatorname{sh} y \sin x=\frac{2 q}{p^{2}+q^{2}+r^{2}+t^{2}-1}, \\
\operatorname{ch} w \operatorname{ch} z \operatorname{ch} y=\frac{p^{2}+q^{2}+r^{2}+t^{2}+1}{p^{2}+q^{2}+r^{2}+t^{2}-1}, \quad \operatorname{ch} w \operatorname{sh} z=\frac{2 r}{p^{2}+q^{2}+r^{2}+t^{2}-1}, \\
\operatorname{sh} w=\frac{2 t}{p^{2}+q^{2}+r^{2}+t^{2}-1} .
\end{gathered}
$$

Тогда

$$
l(A, B)=\frac{\left(p_{A}-p_{B}\right)^{2}+\left(q_{A}-q_{B}\right)^{2}+\left(r_{A}-r_{B}\right)^{2}+\left(t_{A}-t_{B}\right)^{2}}{\left(p_{A}^{2}+q_{A}^{2}+r_{A}^{2}+t_{A}^{2}-1\right)\left(p_{B}^{2}+q_{B}^{2}+r_{B}^{2}+t_{B}^{2}-1\right)} .
$$

Функция (59) задает геометрию де Ситтера в проективной интерпретации, т.е. геометрию на однополостном гиперболоиде в пятимерном псевдоевклидовом пространстве сигнатуры $(+,+,+,+,-)$, задаваемом уравнением $\alpha_{1}^{2}+\alpha_{2}^{2}+\alpha_{3}^{2}+\alpha_{4}^{2}-\alpha_{5}^{2}=1$. Переходим к функции $l(A, B)=(1-f(A, B)) / 2$. Введем стереографические координаты

$$
\begin{gathered}
\operatorname{sh} w \operatorname{sh} z \operatorname{sh} y \cos x=\frac{2 p}{p^{2}+q^{2}+r^{2}-t^{2}+1}, \quad \operatorname{sh} w \operatorname{sh} z \operatorname{sh} y \sin x=\frac{2 q}{p^{2}+q^{2}+r^{2}-t^{2}+1}, \\
\operatorname{sh} w \operatorname{sh} z \operatorname{ch} y=\frac{2 t}{p^{2}+q^{2}+r^{2}-t^{2}+1}, \quad \operatorname{sh} w \operatorname{ch} z=\frac{2 r}{p^{2}+q^{2}+r^{2}-t^{2}+1}, \\
\operatorname{ch} w=\frac{p^{2}+q^{2}+r^{2}-t^{2}-1}{p^{2}+q^{2}+r^{2}-t^{2}+1} .
\end{gathered}
$$

Тогда

$$
l(A, B)=\frac{\left(p_{A}-p_{B}\right)^{2}+\left(q_{A}-q_{B}\right)^{2}+\left(r_{A}-r_{B}\right)^{2}-\left(t_{A}-t_{B}\right)^{2}}{\left(p_{A}^{2}+q_{A}^{2}+r_{A}^{2}-t_{A}^{2}+1\right)\left(p_{B}^{2}+q_{B}^{2}+r_{B}^{2}-t_{B}^{2}+1\right)} .
$$

Функция (60) задает антидеситтерову геометрию в проективной интерпретации, т.е. геометрию на поверхности в пятимерном псевдоевклидовом пространстве сигнатуры $(+,+,+,-,-)$, задаваемом уравнением $\alpha_{1}^{2}+\alpha_{2}^{2}+\alpha_{3}^{2}-\alpha_{4}^{2}-\alpha_{5}^{2}=1$. Переходим к функции $l(A, B)=-(1+f(A, B)) / 2$. Стереографические координаты:

$$
\begin{gathered}
\operatorname{ch} w \operatorname{sh} z \operatorname{sh} y \operatorname{ch} x=\frac{2 p}{p^{2}+q^{2}-r^{2}-t^{2}-1}, \quad \operatorname{ch} w \operatorname{sh} z \operatorname{sh} y \operatorname{sh} x=\frac{2 q}{p^{2}+q^{2}-r^{2}-t^{2}-1}, \\
\operatorname{ch} w \operatorname{sh} z \operatorname{ch} y=\frac{2 t}{p^{2}+q^{2}-r^{2}-t^{2}-1}, \quad \operatorname{ch} w \operatorname{ch} z=\frac{2 r}{p^{2}+q^{2}+r^{2}-t^{2}-1}, \\
\operatorname{sh} w=\frac{p^{2}+q^{2}+r^{2}-t^{2}+1}{p^{2}+q^{2}+r^{2}-t^{2}-1} .
\end{gathered}
$$

Тогда

$$
l(A, B)=\frac{\left(p_{A}-p_{B}\right)^{2}+\left(q_{A}-q_{B}\right)^{2}+\left(r_{A}-r_{B}\right)^{2}-\left(t_{A}-t_{B}\right)^{2}}{\left(p_{A}^{2}+q_{A}^{2}+r_{A}^{2}-t_{A}^{2}-1\right)\left(p_{B}^{2}+q_{B}^{2}+r_{B}^{2}-t_{B}^{2}-1\right)} .
$$

Функция (61) задает геометрию де Ситтера 2-го рода в проективной интерпретации, т.е. геометрию на поверхности в пятимерном псевдоевклидовом пространстве сигнатуры $(+,+,+,-,-)$, задаваемом уравнением $\alpha_{1}^{2}+\alpha_{2}^{2}-\alpha_{3}^{2}-\alpha_{4}^{2}-\alpha_{5}^{2}=1$. Переходим от которой к функции $l(A, B)=$ $(1+f(A, B)) / 2$. Введем новые координаты, используя стереографическую проекцию:

$$
\begin{gathered}
\operatorname{ch} w \operatorname{sh} z \operatorname{sh} y \operatorname{ch} x=\frac{2 p}{p^{2}+q^{2}-r^{2}-t^{2}+1}, \quad \operatorname{ch} w \operatorname{sh} z \operatorname{sh} y \operatorname{sh} x=\frac{2 r}{p^{2}+q^{2}-r^{2}-t^{2}+1}, \\
\operatorname{ch} w \operatorname{sh} z \operatorname{ch} y=\frac{2 t}{p^{2}+q^{2}-r^{2}-t^{2}+1}, \quad \operatorname{ch} w \operatorname{ch} z=\frac{2 q}{p^{2}+q^{2}-r^{2}-t^{2}+1}, \\
\operatorname{sh} w=\frac{p^{2}+q^{2}-r^{2}-t^{2}-1}{p^{2}+q^{2}-r^{2}-t^{2}+1} .
\end{gathered}
$$


Поэтому

$$
l(A, B)=\frac{\left(p_{A}-p_{B}\right)^{2}+\left(q_{A}-q_{B}\right)^{2}-\left(r_{A}-r_{B}\right)^{2}-\left(t_{A}-t_{B}\right)^{2}}{\left(p_{A}^{2}+q_{A}^{2}-r_{A}^{2}-t_{A}^{2}+1\right)\left(p_{B}^{2}+q_{B}^{2}-r_{B}^{2}-t_{B}^{2}+1\right)} .
$$

Функция (62) задает евклидову геометрию, функция (63) - псевдоевклидову геометрию сигнатурой $(+,+,+,-)$, функция $(64)$ - псевдоевклидову геометрию сигнатурой $(+,+,+,-)$, функция (65) - особое расширение евклидовой геометрии, функция (66) - особое расширение псевдоевклидовой геометрии и, наконец, функция (67) - симплектическую геометрию.

В функции пары точек (69) переходим к новым координатам:

$$
\begin{aligned}
& p=\frac{2 x}{(1+w)^{2}+x^{2}+y^{2}+z^{2}}, \quad q=\frac{2 y}{(1+w)^{2}+x^{2}+y^{2}+z^{2}}, \\
& r=\frac{2 z}{(1+w)^{2}+x^{2}+y^{2}+z^{2}}, \quad t=\frac{1-x^{2}-y^{2}-z^{2}-w^{2}}{(1+w)^{2}+x^{2}+y^{2}+z^{2}}
\end{aligned}
$$

при этом

$$
p^{2}+q^{2}+r^{2}+t^{2}-1=\frac{-4 w}{(1+w)^{2}+x^{2}+y^{2}+z^{2}} .
$$

Тогда (69) примет вид

$$
l(A, B)=\frac{\left(x_{A}-x_{B}\right)^{2}+\left(y_{A}-y_{B}\right)^{2}+\left(z_{A}-z_{B}\right)^{2}+\left(w_{A}-w_{B}\right)^{2}}{4 w_{A} w_{B}},
$$

В функции (70) введем координаты

причем

$$
\begin{aligned}
& p=\frac{2 x}{(1+w)^{2}-x^{2}-y^{2}-z^{2}}, \quad q=\frac{2 y}{(1+w)^{2}-x^{2}-y^{2}-z^{2}}, \\
& r=\frac{2 z}{(1+w)^{2}-x^{2}-y^{2}-z^{2}}, \quad t=\frac{1+x^{2}+y^{2}+z^{2}-w^{2}}{(1+w)^{2}-x^{2}-y^{2}-z^{2}},
\end{aligned}
$$

Тогда для (70) имеем

$$
p^{2}+q^{2}+r^{2}-t^{2}+1=\frac{4 w}{(1+w)^{2}-x^{2}-y^{2}-z^{2}} .
$$

$$
l(A, B)=\frac{\left(x_{A}-x_{B}\right)^{2}+\left(y_{A}-y_{B}\right)^{2}+\left(z_{A}-z_{B}\right)^{2}-\left(w_{A}-w_{B}\right)^{2}}{4 w_{A} w_{B}},
$$

Функции (71) запишем в координатах

причем

$$
\begin{aligned}
& p=\frac{2 x}{-(1+w)^{2}-x^{2}-y^{2}+z^{2}}, \quad q=\frac{2 y}{-(1+w)^{2}-x^{2}-y^{2}+z^{2}}, \\
& t=\frac{2 z}{-(1+w)^{2}-x^{2}-y^{2}+z^{2}}, \quad r=\frac{-1+x^{2}+y^{2}-z^{2}+w^{2}}{-(1+w)^{2}-x^{2}-y^{2}+z^{2}},
\end{aligned}
$$

$$
p^{2}+q^{2}+r^{2}-t^{2}+1=\frac{4 w}{-(1+w)^{2}-x^{2}-y^{2}+z^{2}} .
$$

Тогда функция пары точек (71) примет вид

$$
l(A, B)=\frac{\left(x_{A}-x_{B}\right)^{2}+\left(y_{A}-y_{B}\right)^{2}-\left(z_{A}-z_{B}\right)^{2}+\left(w_{A}-w_{B}\right)^{2}}{4 w_{A} w_{B}},
$$

В функции пары точек (72) переходим к новым координатам

$$
\begin{array}{ll}
p=\frac{2 x}{(1+w)^{2}-x^{2}-y^{2}+z^{2}}, & q=\frac{2 y}{(1+w)^{2}-x^{2}-y^{2}+z^{2}}, \\
t=\frac{2 z}{(1+w)^{2}-x^{2}-y^{2}+z^{2}}, & \quad r=\frac{1+x^{2}+y^{2}-z^{2}-w^{2}}{(1+w)^{2}-x^{2}-y^{2}+z^{2}}
\end{array}
$$


при этом

$$
p^{2}+q^{2}-r^{2}-t^{2}+1=\frac{4 w}{(1+w)^{2}-x^{2}-y^{2}+z^{2}} .
$$

Тогда функция пары точек (72) примет вид

$$
l(A, B)=\frac{\left(x_{A}-x_{B}\right)^{2}+\left(y_{A}-y_{B}\right)^{2}-\left(z_{A}-z_{B}\right)^{2}-\left(w_{A}-w_{B}\right)^{2}}{4 w_{A} w_{B}} .
$$

От функций пары точек (57)-(61) удобно перейти к следующим функциям:

$$
\begin{aligned}
& f(A, B)=m_{A} m_{B}+n_{A} n_{B}+l_{A} l_{B}+k_{A} k_{B}+j_{A} j_{B}, m^{2}+n^{2}+l^{2}+k^{2}+j^{2}>0 ; \\
& f(A, B)=m_{A} m_{B}+n_{A} n_{B}-l_{A} l_{B}+k_{A} k_{B}+j_{A} j_{B} B, m^{2}+n^{2}-l^{2}+k^{2}+j^{2}<0 ; \\
& f(A, B)=m_{A} m_{B}+n_{A} n_{B}-l_{A} l_{B}+k_{A} k_{B}+j_{A} j_{B}, m^{2}+n^{2}-l^{2}+k^{2}+j^{2}>0 ; \\
& f(A, B)=m_{A} m_{B}+n_{A} n_{B}-l_{A} l_{B}+k_{A} k_{B}-j_{A} j_{B}, m^{2}+n^{2}-l^{2}+k^{2}-j^{2}>0 ; \\
& f(A, B)=m_{A} m_{B}-n_{A} n_{B}-l_{A} l_{B}+k_{A} k_{B}-j_{A} j_{B}, m^{2}-n^{2}-l^{2}+k^{2}-j^{2}>0 .
\end{aligned}
$$

Заметим, что на сфере функции пары точек (57) и (77) совпадают; группы движений, сохраняющие эти функции, также совпадают. Подобный вывод справедлив и для функция пар точек (58) и (78), для функций (59) и (79), для функций (60) и (80), а также для функций (61) и (81).

4. Основные результаты. В данном пункте приводятся результаты решения задачи вложения для двумерных и трехмерных геометрий локальной максимальной подвижности.

Сформулируем теорему вложения для двумерных геометрий локальной максимальной подвижности.

Теорема 1. Рассмотрим произвольную точку $C \in M$ и ее координатную окрестность $U$, $\operatorname{dim} M=3$. Возъмем также такие две точки $A, B \in U$ с окрестностями $U(A) u U(B)$, ито $U(A) \cup U(B) \subset U$, причем $\langle A, B\rangle,\left\langle A^{\prime}, B^{\prime}\right\rangle \in S_{f}$ при любых $A^{\prime} \in U(A)$ u $\forall B^{\prime} \in U(B)$. Тогда функиия пары точек (2) в аналитическом многообразии $M$, являющаяся двухточечным инвариантом шестипараметрической группы движений, в окрестности $U(A) \times U(B)$ в подходящих локальных координатах и масштабном преобразовании $(\varphi(f) \rightarrow f)$ имеет один из следующих видов:

(i) для функиии $\theta$ вида (28): (53), (54), (55), (35);

(ii) для функиии $\theta$ вида (29): (54), (55), (56), (36);

(iii) для функиии $\theta$ вида (30): (54), (55), (56), (36);

(iv) для функиии $\theta$ вида (13): (35), (36), (41), (50), (51);

(v) для функиии $\theta$ вида (14): (36), (42), (52);

(vi) для функиии $\theta$ вида (15): (37);

(vii) для функиии $\theta$ вида (16): (38), (39), (42);

(viii) для функиии $\theta$ вида (17): (43);

(ix) для функиии $\theta$ вида (18): (44);

(x) для функиии $\theta$ вида (19): (45);

(xi) для бункици $\theta$ вида (20): (41);

(xii) для функици $\theta$ вида (21): (41);

(xiii) для функиии $\theta$ вида (22): (42).

Для доказательства этой теоремы, как сказано выше, записываются функциональные уравнения специального вида, которые получаются при подстановке формул (3), (4) или

$$
X=X_{1} \partial_{m}+X_{2} \partial_{n}+X_{3} \partial_{l}+P \partial_{k}
$$

в условие локальной инвариантности (2). В явном виде эти формулы записываются следующим образом: 
(i) для функции пары точек (28):

$$
\begin{aligned}
{\left[m_{A} X_{1}(B)+m_{B} X_{1}(A)+n_{A} X_{2}(B)+n_{B} X_{2}(A)+l_{A} X_{3}(B)+l_{B} X_{3}(A)\right] \frac{\partial \chi}{\partial \theta}+} & \\
+\left(P(A) \frac{\partial \chi}{\partial k_{A}}+P(B) \frac{\partial \chi}{\partial k_{B}}\right) & =0
\end{aligned}
$$

(ii) для функции пары точек (29):

$$
\begin{aligned}
{\left[m_{A} X_{1}(B)+m_{B} X_{1}(A)+n_{A} X_{2}(B)+n_{B} X_{2}(A)-l_{A} X_{3}(B)-l_{B} X_{3}(A)\right] \frac{\partial \chi}{\partial \theta}+} & \\
+\left(P(A) \frac{\partial \chi}{\partial k_{A}}+P(B) \frac{\partial \chi}{\partial k_{B}}\right) & =0
\end{aligned}
$$

(iii) для функции пары точек (30):

$$
\begin{aligned}
{\left[m_{A} X_{1}(B)+m_{B} X_{1}(A)-n_{A} X_{2}(B)-n_{B} X_{2}(A)-l_{A} X_{3}(B)-l_{B} X_{3}(A)\right] \frac{\partial \chi}{\partial \theta}+} & \\
+\left(P(A) \frac{\partial \chi}{\partial k_{A}}+P(B) \frac{\partial \chi}{\partial k_{B}}\right) & =0
\end{aligned}
$$

(iv) для функции пары точек (13):

$$
\begin{aligned}
2\left[\left(x_{A}-x_{B}\right)\left(X_{1}(A)-X_{1}(B)\right)+\left(y_{A}-y_{B}\right)\left(X_{2}(A)-\right.\right. & \left.\left.X_{2}(B)\right)\right] \frac{\partial \chi}{\partial \theta}+ \\
+\left(P(A) \frac{\partial \chi}{\partial w_{A}}+P(B) \frac{\partial \chi}{\partial w_{B}}\right) & =0
\end{aligned}
$$

(v) для функции пары точек (14):

$$
\begin{aligned}
2\left[\left(x_{A}-x_{B}\right)\left(X_{1}(A)-X_{1}(B)\right)-\left(y_{A}-y_{B}\right)\left(X_{2}(A)-\right.\right. & \left.\left.X_{2}(B)\right)\right] \frac{\partial \chi}{\partial \theta}+ \\
& +\left(P(A) \frac{\partial \chi}{\partial w_{A}}+P(B) \frac{\partial \chi}{\partial w_{B}}\right)=0
\end{aligned}
$$

(vi) для функции пары точек (15):

$$
\left[y_{B}\left(X_{1}(A)-y_{A} X_{1}(B)-x_{B} X_{2}(A)+x_{A} X_{2}(B)\right] \frac{\partial \chi}{\partial \theta}+\left(P(A) \frac{\partial \chi}{\partial w_{A}}+P(B) \frac{\partial \chi}{\partial w_{B}}\right)=0\right.
$$

(vii) для функции пары точек (16):

$$
\begin{aligned}
& {\left[\left(x_{A}-x_{B}\right)\left(X_{2}(A)-X_{2}(B)\right)-\left(y_{A}-y_{B}\right)\right.}\left.\left(X_{1}(A)-X_{1}(B)\right)\right] \frac{\partial \chi}{\partial \theta}+ \\
&+\left(y_{A}-y_{B}\right)^{2}\left(P(A) \frac{\partial \chi}{\partial w_{A}}+P(B) \frac{\partial \chi}{\partial w_{B}}\right)=0
\end{aligned}
$$

(viii) для функции пары точек (17):

$$
\begin{gathered}
2\left[\left(\left(x_{A}-x_{B}\right)-\gamma\left(y_{A}-y_{B}\right)\right)\left(X_{1}(A)-X_{1}(B)\right)+\left(\left(y_{A}-y_{B}\right)+\gamma\left(x_{A}-x_{B}\right)\right)\left(X_{2}(A)-X_{2}(B)\right)\right] \times \\
\times \frac{\partial \chi}{\partial \theta}+e^{-2 \gamma \operatorname{arctg} \frac{y_{A}-y_{B}}{x_{A}-x_{B}}}\left(P(A) \frac{\partial \chi}{\partial w_{A}}+P(B) \frac{\partial \chi}{\partial w_{B}}\right)=0,
\end{gathered}
$$

(ix) для функции пары точек (18):

$$
\begin{aligned}
{\left[\beta\left(x_{A}-x_{B}\right)\left(X_{2}(A)-X_{2}(B)\right)+\right.} & \left.\varepsilon\left(y_{A}-y_{B}\right)\left(X_{1}(A)-X_{1}(B)\right)\right] \frac{\partial \chi}{\partial \theta}+ \\
& +\left(x_{A}-x_{B}\right)\left(y_{A}-y_{B}\right)\left(P(A) \frac{\partial \chi}{\partial w_{A}}+P(B) \frac{\partial \chi}{\partial w_{B}}\right)=0
\end{aligned}
$$


(х) для функции пары точек (19):

$$
\begin{aligned}
2\left[( ( x _ { A } - x _ { B } ) - ( y _ { A } - y _ { B } ) ) \left(X_{1}(A)\right.\right. & \left.\left.-X_{1}(B)\right)+\left(x_{A}-x_{B}\right)\left(X_{2}(A)-X_{2}(B)\right)\right] \frac{\partial \chi}{\partial \theta}+ \\
& +\exp \left(-2 \frac{y_{A}-y_{B}}{x_{A}-x_{B}}\right)\left(P(A) \frac{\partial \chi}{\partial w_{A}}+P(B) \frac{\partial \chi}{\partial w_{B}}\right)=0 .
\end{aligned}
$$

(xi) для функции пары точек (20):

$$
\begin{aligned}
& 2\left[\frac{\left(x_{A}-x_{B}\right)\left(X_{1}(A)-X_{1}(B)\right)+\left(y_{A}-y_{B}\right)\left(X_{2}(A)-X_{2}(B)\right)}{\left(x_{A}^{2}+y_{A}^{2}+1\right)\left(x_{B}^{2}+y_{B}^{2}+1\right)}-\right. \\
& -\frac{\left(x_{A}-x_{B}\right)^{2}+\left(y_{A}-y_{B}\right)^{2}}{\left(x_{A}^{2}+y_{A}^{2}+1\right)^{2}\left(x_{B}^{2}+y_{B}^{2}+1\right)}\left(x_{A} X_{1}(A)+y_{A} X_{2}(A)\right)- \\
& \left.-\frac{\left(x_{A}-x_{B}\right)^{2}+\left(y_{A}-y_{B}\right)^{2}}{\left(x_{A}^{2}+y_{A}^{2}+1\right)\left(x_{B}^{2}+y_{B}^{2}+1\right)^{2}}\left(x_{B} X_{1}(B)+y_{B} X_{2}(B)\right)\right]+\left(P(A) \frac{\partial \chi}{\partial w_{A}}+P(B) \frac{\partial \chi}{\partial w_{B}}\right)=0,
\end{aligned}
$$

(xii) для функции пары точек (21):

$$
\begin{aligned}
& 2\left[\frac{\left(x_{A}-x_{B}\right)\left(X_{1}(A)-X_{1}(B)\right)+\left(y_{A}-y_{B}\right)\left(X_{2}(A)-X_{2}(B)\right)}{\left(x_{A}^{2}+y_{A}^{2}-1\right)\left(x_{B}^{2}+y_{B}^{2}-1\right)}-\right. \\
& -\frac{\left(x_{A}-x_{B}\right)^{2}+\left(y_{A}-y_{B}\right)^{2}}{\left(x_{A}^{2}+y_{A}^{2}-1\right)^{2}\left(x_{B}^{2}+y_{B}^{2}-1\right)}\left(x_{A} X_{1}(A)+y_{A} X_{2}(A)\right)- \\
& \left.-\frac{\left(x_{A}-x_{B}\right)^{2}+\left(y_{A}-y_{B}\right)^{2}}{\left(x_{A}^{2}+y_{A}^{2}-1\right)\left(x_{B}^{2}+y_{B}^{2}-1\right)^{2}}\left(x_{B} X_{1}(B)+y_{B} X_{2}(B)\right)\right]+\left(P(A) \frac{\partial \chi}{\partial w_{A}}+P(B) \frac{\partial \chi}{\partial w_{B}}\right)=0,
\end{aligned}
$$

(xiii) для функции пары точек $(22)$ :

$$
\begin{aligned}
& 2\left[\frac{\left(x_{A}-x_{B}\right)\left(X_{1}(A)-X_{1}(B)\right)-\left(y_{A}-y_{B}\right)\left(X_{2}(A)-X_{2}(B)\right)}{\left(x_{A}^{2}-y_{A}^{2}-1\right)\left(x_{B}^{2}-y_{B}^{2}-1\right)}-\right. \\
& \quad-\frac{\left(x_{A}-x_{B}\right)^{2}-\left(y_{A}-y_{B}\right)^{2}}{\left(x_{A}^{2}-y_{A}^{2}-1\right)^{2}\left(x_{B}^{2}-y_{B}^{2}-1\right)}\left(x_{A} X_{1}(A)-y_{A} X_{2}(A)\right)- \\
& \left.\quad-\frac{\left(x_{A}-x_{B}\right)^{2}-\left(y_{A}-y_{B}\right)^{2}}{\left(x_{A}^{2}-y_{A}^{2}-1\right)\left(x_{B}^{2}-y_{B}^{2}-1\right)^{2}}\left(x_{B} X_{1}(B)-y_{B} X_{2}(B)\right)\right]+\left(P(A) \frac{\partial \chi}{\partial w_{A}}+P(B) \frac{\partial \chi}{\partial w_{B}}\right)=0 .
\end{aligned}
$$

Эти функциональные уравнения решаются аналитически, для чего неизвестные (компоненты оператора алгебры Ли и функция $\chi$ ) представляются рядами Тейлора (7)-(9), а также учитываются условия невырожденности (3). Подстановка этих разложений в выше выписанные функциональные уравнения приводит к задаче перебора коэффициентов рядов Тейлора, которая существенно упрощается с применением математического пакета программ Maple 17 (см. [5]). Заметим, что при записи функциональных уравнений для функций пар точек (20)-(22) координаты $p$ и $q$ переобозначены через $x$ и $y$.

Теперь сформулируем теорему вложения для трехмерных геометрий локальной максимальной подвижности.

Теорема 2. Рассмотрим произвольную точку $C \in M$ и ее координатную окрестность $U$, $\operatorname{dim} M=4$. Возвмем также две точки $A, B \in U$ с окрестностями $U(A)$ и $U(B)$ такие, что $U(A) \cup U(B) \subset U$, причем $\langle A, B\rangle,\left\langle A^{\prime}, B^{\prime}\right\rangle \in S_{f}, \forall A^{\prime} \in U(A), \forall B^{\prime} \in U(B)$. Тогда фучкиия (2) в аналитическом многообразии $M$, являющаяся двухточечным инвариантом десятипараметрической группы движений, в окрестности $U(A) \times U(B)$ в подходящих локальных координатах и масштабном преобразовании $(\varphi(f) \rightarrow f)$ имеет один из следующих видов:

(i) для функции $\theta$ вида (53): (77), (78), (79), (62), (63);

(ii) для функиии $\theta$ вида (54): (78), (79), (80), (81), (63), (64);

(iii) для функции $\theta$ вида (55): (78), (79), (80), (81), (63), (64);

(iv) для функиии $\theta$ вида (56): (80), (81), (64); 
(v) для функиии $\theta$ вида (35): (62), (63), (65), (73), (74);

(vi) для функиии $\theta$ вида (36): (63), (64), (66), (75), (76);

(vii) для функиии $\theta$ вида (37): (67);

(viii) для функиии $\theta$ вида (38)-(45): нет;

(ix) для функиии $\theta$ вида (46): (65);

(x) для функиии $\theta$ вида (47): (65);

(xi) для функиии $\theta$ вида (48): (66);

(xii) для функиии $\theta$ вида (49): (66).

Для доказательства теоремы, как сказано выше, записываются функциональные уравнения специального вида, которые получаются при подстановке формул (3), (5) или

$$
X=X_{1} \partial_{m}+X_{2} \partial_{n}+X_{3} \partial_{l}+X_{4} \partial_{k}+P \partial_{j}
$$

в условие локальной инвариантности (2). В явном виде они имеют следующую запись:

(i) для функции пары точек (53):

$$
\begin{aligned}
{\left[m_{A} X_{1}(B)+m_{B} X_{1}(A)+\right.} & n_{A} X_{2}(B)+n_{B} X_{2}(A)+l_{A} X_{3}(B)+l_{B} X_{3}(A)+ \\
& \left.+k_{A} X_{4}(B)+k_{B} X_{4}(A)\right] \frac{\partial \chi}{\partial \theta}+\left(P(A) \frac{\partial \chi}{\partial j_{A}}+P(B) \frac{\partial \chi}{\partial j_{B}}\right)=0,
\end{aligned}
$$

(ii) для функции пары точек (54):

$$
\begin{aligned}
{\left[m_{A} X_{1}(B)+m_{B} X_{1}(A)+\right.} & n_{A} X_{2}(B)+n_{B} X_{2}(A)-l_{A} X_{3}(B)-l_{B} X_{3}(A)+ \\
& \left.+k_{A} X_{4}(B)+k_{B} X_{4}(A)\right] \frac{\partial \chi}{\partial \theta}+\left(P(A) \frac{\partial \chi}{\partial j_{A}}+P(B) \frac{\partial \chi}{\partial j_{B}}\right)=0,
\end{aligned}
$$

(iii) для функции пары точек (55):

$$
\begin{aligned}
{\left[m_{A} X_{1}(B)+m_{B} X_{1}(A)+\right.} & n_{A} X_{2}(B)+n_{B} X_{2}(A)-l_{A} X_{3}(B)-l_{B} X_{3}(A)+ \\
& \left.+k_{A} X_{4}(B)+k_{B} X_{4}(A)\right] \frac{\partial \chi}{\partial \theta}+\left(P(A) \frac{\partial \chi}{\partial j_{A}}+P(B) \frac{\partial \chi}{\partial j_{B}}\right)=0
\end{aligned}
$$

(iv) для функции пары точек (56):

$$
\begin{aligned}
& {\left[m_{A} X_{1}(B)+m_{B} X_{1}(A)-n_{A} X_{2}(B)-n_{B} X_{2}(A)-l_{A} X_{3}(B)-l_{B} X_{3}(A)+\right.} \\
& \left.\quad+k_{A} X_{4}(B)+k_{B} X_{4}(A)\right] \frac{\partial \chi}{\partial \theta}+\left(P(A) \frac{\partial \chi}{\partial j_{A}}+P(B) \frac{\partial \chi}{\partial j_{B}}\right)=0
\end{aligned}
$$

(v) для функции пары точек (35):

$$
\begin{aligned}
& 2\left[\left(x_{A}-x_{B}\right)\left(X_{1}(A)-X_{1}(B)\right)+\left(y_{A}-y_{B}\right)\left(X_{2}(A)-X_{2}(B)\right)+\right. \\
& \left.\quad+\left(z_{A}-z_{B}\right)\left(X_{3}(A)-X_{3}(B)\right)\right] \frac{\partial \chi}{\partial \theta}+\left(P(A) \frac{\partial \chi}{\partial w_{A}}+P(B) \frac{\partial \chi}{\partial w_{B}}\right)=0,
\end{aligned}
$$

(vi) для функции пары точек (36):

$$
\begin{aligned}
2\left[( x _ { A } - x _ { B } ) \left(X_{1}(A)\right.\right. & \left.-X_{1}(B)\right)+\left(y_{A}-y_{B}\right)\left(X_{2}(A)-X_{2}(B)\right)- \\
& \left.-\left(z_{A}-z_{B}\right)\left(X_{3}(A)-X_{3}(B)\right)\right] \frac{\partial \chi}{\partial \theta}+\left(P(A) \frac{\partial \chi}{\partial w_{A}}+P(B) \frac{\partial \chi}{\partial w_{B}}\right)=0,
\end{aligned}
$$


(vii) для функции пары точек (37):

$$
\begin{aligned}
{\left[y_{B}\left(X_{1}(A)-y_{A} X_{1}(B)-x_{B} X_{2}(A)+x_{A} X_{2}(B)+X_{3}(A)-X_{3}(B)\right] \frac{\partial \chi}{\partial \theta}+\right.} & \\
+\left(P(A) \frac{\partial \chi}{\partial w_{A}}+P(B) \frac{\partial \chi}{\partial w_{B}}\right) & =0
\end{aligned}
$$

(viii) для функции пары точек (38):

$$
\begin{aligned}
& {\left[\left(x_{A}-x_{B}\right)\left(X_{2}(A)-X_{2}(B)\right)-\left(y_{A}-y_{B}\right)\left(X_{1}(A)-X_{1}(B)\right)+\right.} \\
& \left.\quad+\left(x_{A}-x_{B}\right)^{2}\left(X_{3}(A)+X_{3}(B)\right)\right] \frac{\partial \chi}{\partial \theta}+\left(x_{A}-x_{B}\right)^{2}\left(P(A) \frac{\partial \chi}{\partial w_{A}}+P(B) \frac{\partial \chi}{\partial w_{B}}\right)=0
\end{aligned}
$$

(ix) для функции пары точек (39):

$$
\begin{aligned}
& {\left[\left(x_{A}-x_{B}\right)\left(X_{2}(A)-X_{2}(B)\right)-\left(y_{A}-y_{B}\right)\left(X_{1}(A)-X_{1}(B)\right)+\right.} \\
& \left.+\left(x_{A}-x_{B}\right)^{2}\left(X_{3}(A)+X_{3}(B)\right)\right] \frac{\partial \chi}{\partial \theta}+ \\
& \quad+\left(x_{A}-x_{B}\right)^{2} e^{-z_{A}-z_{B}}\left(P(A) \frac{\partial \chi}{\partial w_{A}}+P(B) \frac{\partial \chi}{\partial w_{B}}\right)=0
\end{aligned}
$$

(х) для функции пары точек (40):

$$
\begin{aligned}
& {\left[\left(x_{A}-x_{B}\right)\left(X_{2}(A)-X_{2}(B)\right)-\left(y_{A}-y_{B}\right)\left(X_{1}(A)-X_{1}(B)\right)+\right.} \\
& \left.+\left[\left(x_{A}-x_{B}\right)^{2}+\left(y_{A}-y_{B}\right)^{2}\right]\left(X_{3}(A)+X_{3}(B)\right)\right] \frac{\partial \chi}{\partial \theta} \\
& \quad+\left[\left(x_{A}-x_{B}\right)^{2}+\left(y_{A}-y_{B}\right)^{2}\right]\left(P(A) \frac{\partial \chi}{\partial w_{A}}+P(B) \frac{\partial \chi}{\partial w_{B}}\right)=0
\end{aligned}
$$

(xi) для функции пары точек (41):

$$
\begin{aligned}
2\left[\left(x_{A}-x_{B}\right)\left(X_{1}(A)-X_{1}(B)\right)+\left(y_{A}-y_{B}\right)\right. & \left(X_{2}(A)-X_{2}(B)\right)+ \\
+\left[\left(x_{A}-x_{B}\right)^{2}+\left(y_{A}-y_{B}\right)^{2}\right] & \left.\left(X_{3}(A)+X_{3}(B)\right)\right] \frac{\partial \chi}{\partial \theta}+ \\
& +e^{-2 z_{A}-2 z_{B}}\left(P(A) \frac{\partial \chi}{\partial w_{A}}+P(B) \frac{\partial \chi}{\partial w_{B}}\right)=0
\end{aligned}
$$

(xii) для функции пары точек (42):

$$
\begin{aligned}
& 2\left[\left(x_{A}-x_{B}\right)\left(X_{1}(A)-X_{1}(B)\right)-\left(y_{A}-y_{B}\right)\right.\left(X_{2}(A)-X_{2}(B)\right)+ \\
&\left.+\left[\left(x_{A}-x_{B}\right)^{2}-\left(y_{A}-y_{B}\right)^{2}\right]\left(X_{3}(A)+X_{3}(B)\right)\right] \frac{\partial \chi}{\partial \theta}+ \\
& \quad+e^{-2 z_{A}-2 z_{B}}\left(P(A) \frac{\partial \chi}{\partial w_{A}}+P(B) \frac{\partial \chi}{\partial w_{B}}\right)=0
\end{aligned}
$$

(xiii) для функции пары точек (43):

$$
\begin{gathered}
2\left[\left(\left(x_{A}-x_{B}\right)-\gamma\left(y_{A}-y_{B}\right)\right)\left(X_{1}(A)-X_{1}(B)\right)+\left(\left(y_{A}-y_{B}\right)+\gamma\left(x_{A}-x_{B}\right)\right)\left(X_{2}(A)-X_{2}(B)\right)+\right. \\
\left.+\left[\left(x_{A}-x_{B}\right)^{2}+\left(y_{A}-y_{B}\right)^{2}\right]\left(X_{3}(A)+X_{3}(B)\right)\right] \frac{\partial \chi}{\partial \theta}+ \\
\quad+\exp \left(-2 \gamma \operatorname{arctg} \frac{y_{A}-y_{B}}{x_{A}-x_{B}}\right)\left(P(A) \frac{\partial \chi}{\partial w_{A}}+P(B) \frac{\partial \chi}{\partial w_{B}}\right)=0
\end{gathered}
$$


(xiv) для функции пары точек (44):

$$
\begin{aligned}
& {\left[\beta ( x _ { A } - x _ { B } ) \left(X_{2}(A)-\right.\right.}\left.X_{2}(B)\right)+ \\
&+\left(x_{A}-x_{B}\right)\left(y_{A}-y_{B}\right)\left(X_{1}(A)-X_{1}(B)\right)+ \\
& \quad+\left(x_{A}-x_{B}\right)\left(y_{A}-y_{B}\right)\left(P(A) \frac{\partial \chi}{\partial w_{A}}+P(B) \frac{\partial \chi}{\partial w_{B}}\right)=0
\end{aligned}
$$

(xv) для функции пары точек (45):

$$
\begin{aligned}
& 2\left[\left(\left(x_{A}-x_{B}\right)-\left(y_{A}-y_{B}\right)\right)\left(X_{1}(A)-X_{1}(B)\right)+\left(x_{A}-x_{B}\right)\left(X_{2}(A)-X_{2}(B)\right)+\right. \\
& \left.+\left(x_{A}-x_{B}\right)^{2}\left(X_{3}(A)+X_{3}(B)\right)\right] \frac{\partial \chi}{\partial \theta}+ \\
& \quad+\exp \left(-2 \frac{y_{A}-y_{B}}{x_{A}-x_{B}}\right)\left(P(A) \frac{\partial \chi}{\partial w_{A}}+P(B) \frac{\partial \chi}{\partial w_{B}}\right)=0
\end{aligned}
$$

(xvi) для функции пары точек (46):

$$
\begin{gathered}
2\left[\frac{\left(x_{A}-x_{B}\right)\left(X_{1}(A)-X_{1}(B)\right)+\left(y_{A}-y_{B}\right)\left(X_{2}(A)-X_{2}(B)\right)+\left(z_{A}-z_{B}\right)\left(X_{3}(A)-X_{3}(B)\right)}{\left(x_{A}^{2}+y_{A}^{2}+z_{A}^{2}+1\right)\left(x_{B}^{2}+y_{B}^{2}+z_{B}^{2}+1\right)}-\right. \\
-\frac{\left(x_{A}-x_{B}\right)^{2}+\left(y_{A}-y_{B}\right)^{2}+\left(z_{A}-z_{B}\right)^{2}}{\left(x_{A}^{2}+y_{A}^{2}+z_{A}^{2}+1\right)^{2}\left(x_{B}^{2}+y_{B}^{2}+z_{B}^{2}+1\right)}\left(x_{A} X_{1}(A)+y_{A} X_{2}(A)+z_{A} X_{3}(A)\right)- \\
\left.-\frac{\left(x_{A}-x_{B}\right)^{2}+\left(y_{A}-y_{B}\right)^{2}+\left(z_{A}-z_{B}\right)^{2}}{\left(x_{A}^{2}+y_{A}^{2}+z_{A}^{2}+1\right)\left(x_{B}^{2}+y_{B}^{2}+z_{B}^{2}+1\right)^{2}}\left(x_{B} X_{1}(B)+y_{B} X_{2}(B)+z_{B} X_{3}(B)\right)\right]+ \\
+\left(P(A) \frac{\partial \chi}{\partial w_{A}}+P(B) \frac{\partial \chi}{\partial w_{B}}\right)=0
\end{gathered}
$$

(xvii) для функции пары точек (47):

$$
\begin{gathered}
2\left[\frac{\left(x_{A}-x_{B}\right)\left(X_{1}(A)-X_{1}(B)\right)+\left(y_{A}-y_{B}\right)\left(X_{2}(A)-X_{2}(B)\right)+\left(z_{A}-z_{B}\right)\left(X_{3}(A)-X_{3}(B)\right)}{\left(x_{A}^{2}+y_{A}^{2}+z_{A}^{2}-1\right)\left(x_{B}^{2}+y_{B}^{2}+z_{B}^{2}-1\right)}-\right. \\
-\frac{\left(x_{A}-x_{B}\right)^{2}+\left(y_{A}-y_{B}\right)^{2}+\left(z_{A}-z_{B}\right)^{2}}{\left(x_{A}^{2}+y_{A}^{2}+z_{A}^{2}-1\right)^{2}\left(x_{B}^{2}+y_{B}^{2}+z_{B}^{2}-1\right)}\left(x_{A} X_{1}(A)+y_{A} X_{2}(A)+z_{A} X_{3}(A)\right)- \\
\left.-\frac{\left(x_{A}-x_{B}\right)^{2}+\left(y_{A}-y_{B}\right)^{2}+\left(z_{A}-z_{B}\right)^{2}}{\left(x_{A}^{2}+y_{A}^{2}+z_{A}^{2}-1\right)\left(x_{B}^{2}+y_{B}^{2}+z_{B}^{2}-1\right)^{2}}\left(x_{B} X_{1}(B)+y_{B} X_{2}(B)+z_{B} X_{3}(B)\right)\right]+ \\
+\left(P(A) \frac{\partial \chi}{\partial w_{A}}+P(B) \frac{\partial \chi}{\partial w_{B}}\right)=0,
\end{gathered}
$$

(xviii) для функции пары точек (48):

$$
\begin{gathered}
2\left[\frac{\left(x_{A}-x_{B}\right)\left(X_{1}(A)-X_{1}(B)\right)+\left(y_{A}-y_{B}\right)\left(X_{2}(A)-X_{2}(B)\right)-\left(z_{A}-z_{B}\right)\left(X_{3}(A)-X_{3}(B)\right)}{\left(x_{A}^{2}+y_{A}^{2}-z_{A}^{2}+1\right)\left(x_{B}^{2}-y_{B}^{2}+z_{B}^{2}+1\right)}-\right. \\
-\frac{\left(x_{A}-x_{B}\right)^{2}+\left(y_{A}-y_{B}\right)^{2}-\left(z_{A}-z_{B}\right)^{2}}{\left(x_{A}^{2}+y_{A}^{2}-z_{A}^{2}+1\right)^{2}\left(x_{B}^{2}+y_{B}^{2}-z_{B}^{2}+1\right)}\left(x_{A} X_{1}(A)+y_{A} X_{2}(A)-z_{A} X_{3}(A)\right)- \\
\left.-\frac{\left(x_{A}-x_{B}\right)^{2}+\left(y_{A}-y_{B}\right)^{2}-\left(z_{A}-z_{B}\right)^{2}}{\left(x_{A}^{2}+y_{A}^{2}-z_{A}^{2}+1\right)\left(x_{B}^{2}+y_{B}^{2}-z_{B}^{2}+1\right)^{2}}\left(x_{B} X_{1}(B)+y_{B} X_{2}(B)-z_{B} X_{3}(B)\right)\right]+ \\
+\left(P(A) \frac{\partial \chi}{\partial w_{A}}+P(B) \frac{\partial \chi}{\partial w_{B}}\right)=0
\end{gathered}
$$


(xix) для функции пары точек (49):

$$
\begin{gathered}
2\left[\frac{\left(x_{A}-x_{B}\right)\left(X_{1}(A)-X_{1}(B)\right)+\left(y_{A}-y_{B}\right)\left(X_{2}(A)-X_{2}(B)\right)-\left(z_{A}-z_{B}\right)\left(X_{3}(A)-X_{3}(B)\right)}{\left(x_{A}^{2}+y_{A}^{2}-z_{A}^{2}-1\right)\left(x_{B}^{2}-y_{B}^{2}+z_{B}^{2}-1\right)}-\right. \\
-\frac{\left(x_{A}-x_{B}\right)^{2}+\left(y_{A}-y_{B}\right)^{2}-\left(z_{A}-z_{B}\right)^{2}}{\left(x_{A}^{2}+y_{A}^{2}-z_{A}^{2}-1\right)^{2}\left(x_{B}^{2}+y_{B}^{2}-z_{B}^{2}-1\right)}\left(x_{A} X_{1}(A)+y_{A} X_{2}(A)-z_{A} X_{3}(A)\right)- \\
\left.-\frac{\left(x_{A}-x_{B}\right)^{2}+\left(y_{A}-y_{B}\right)^{2}-\left(z_{A}-z_{B}\right)^{2}}{\left(x_{A}^{2}+y_{A}^{2}-z_{A}^{2}-1\right)\left(x_{B}^{2}+y_{B}^{2}-z_{B}^{2}-1\right)^{2}}\left(x_{B} X_{1}(B)+y_{B} X_{2}(B)-z_{B} X_{3}(B)\right)\right]+ \\
+\left(P(A) \frac{\partial \chi}{\partial w_{A}}+P(B) \frac{\partial \chi}{\partial w_{B}}\right)=0 .
\end{gathered}
$$

Выше выписанные функциональные уравнения решаются аналитически, т.е. неизвестные ищутся в виде рядов Тейлора (7)-(9). Это приводит к задаче перебора коэффициентов в разложениях, которая существенно упрощается с применением математического пакета программ Maple 17 (см. [5]). Заметим, что при записи функциональных уравнений для функций пар точек (46)-(49) координаты $p, q, r$ переобозначены через $x, y, z$.

5. Заключение. В данной работе приведены результаты решения задачи вложения двумерных геометрий локальной максимальной подвижности в трехмерные геометрии локальной максимальной подвижности, а также результаты решения задачи вложения трехмерных геометрий локальной максимальной подвижности в четырехмерные геометрии локальной максимальной подвижности. Данная задача может быть продолжена и на более высокие размерности.

Автор выражает глубокую благодарность профессору Г. Г. Михайличенко за поддержку исследований.

\section{СПИСОК ЛИТЕРАТУРЫ}

1. Бердинский Д. А., Тайманов И. А. Поверхности в трехмерных группах Ли// Сиб. мат. ж. - 2005. 46, № 6. - С. $1248-1264$.

2. Бредон Г. Введение в теорию компактных групп преобразований. - М.: Наука, 1980.

3. Громол Д., Клингенберг В., Мейер В. Римонова геометрия в целом. - М.: Мир, 1971.

4. Дубровин Б. А., Новиков С. П., Фоменко А. Т. Современная геометрия: Методы и проложения. - М.: Наука, 1986.

5. Дъяконов В. Maple 10/11/12/13/14 в математических вычислениях. - М.: ДМС, 2014.

6. Кыров В. А. Аналитический метод вложения многомерных псевдоевклидовых геометрий// Сиб. электрон. мат. изв. $-2018 .-15 .-$ С. $741-758$.

7. Къров В. А. Вложение многомерных особых расширений псевдоевклидовых геометрий// Челяб. физ.мат. ж. - 2018. - 4, № 3 . - С. 408-420.

8. Кыров В. А. Функциональные уравнения в псевдоевклидовой геометрии// Сиб. ж. индустр. мат. 2010. - 13, № 4. - C. 38-51.

9. Кыров В. А., Богданова Р. А. Группы движений некоторых трехмерных геометрий максимальной подвижности// Сиб. мат. ж. - 2018. - 59, № 2. - С. 412-421.

10. Кыров В. А., Михайличенко Г. Г. Аналитический метод вложения евклидовой и псевдоевклидовой геометрий// Тр. Ин-та мат. мех. УрО РАН. - 2017. - 23, № 2. - С. 167-181.

11. Кыров В. А., Михайличенко Г. Г. Аналитический метод вложения симплектической геометрии// Сиб. электрон. мат. изв. $-2017 .-14 .-$ С. $657-672$.

12. Лев В. Х. Трехмерные геометрии в теории физических структур// Вычисл. сист. $-1988 .-125$. C. $90-103$.

13. Михайличенко Г. Г. Математические основы и результаты теории физических структур. - ГорноАлтайск: ГАГУ, 2016.

14. Овсянников Л. В. Групповой анализ дифференциальных уравнений. - М.: Наука, 1978.

15. Фихтенгольи Г. М. Курс дифференциального и интегрального исчисления. - М.: Физматгиз, 1963.

16. Mikhailichenko G. G., Borodin A. N. The mathematical basics and results of the theory of physical structures, 2012/ arXiv: 1602.02795v1 [math-ph]. 
17. Thurston W. P. Three-dimensional manifolds, Kleinian groups and hyperbolic geometry// Bull. Am. Math. Soc. - 1982. - 6, № 3. - P. 357-381.

Кыров Владимир Александрович

Горно-Алтайский государственный университет

E-mail: kyrovVA@yandex.ru 